\title{
A Study of Zoledronic Acid as Neo-Adjuvant, Perioperative Therapy in Patients with Resectable Pancreatic Ductal Adenocarcinoma
}

\author{
Dominic E. Sanford ${ }^{1}$, Matthew R. Porembka ${ }^{1}$, Roheena Z. Panni ${ }^{1}$, Jonathan B. Mitchem ${ }^{1}$, \\ Brian A. Belt ${ }^{1}$, Stacey M. Plambeck-Suess ${ }^{1}$, Goldie Lin ${ }^{1}$, David G. DeNardo ${ }^{2}$, Ryan C. Fields ${ }^{1,3}$, \\ William G. Hawkins ${ }^{1,3}$, Steven M. Strasberg ${ }^{1,3}$, Craig Lockhart ${ }^{3,4}$, Andrea Wang-Gillam ${ }^{3,4}$, \\ Simon Peter Goedegebuure ${ }^{1,3}$, David C. Linehan ${ }^{1,3}$
}

\footnotetext{
${ }^{1}$ Department of Surgery, School of Medicine, Washington University, St. Louis, USA; ${ }^{2}$ Department of Medicine, School of Medicine, Washington University, St. Louis, USA; ${ }^{3}$ Alvin J. Siteman Cancer Center, St. Louis, USA; ${ }^{4}$ Division of Oncology, Department of Medical Oncology, School of Medicine, Washington University, St. Louis, USA.

Email: linehand@wudosis.wustl.edu
}

Received February 20 2 th 2013 ; revised March $22^{\text {nd }}, 2013$; accepted March $31^{\text {st }}, 2013$

Copyright (C) 2013 Dominic E. Sanford et al. This is an open access article distributed under the Creative Commons Attribution License, which permits unrestricted use, distribution, and reproduction in any medium, provided the original work is properly cited.

\begin{abstract}
Background: Pancreatic ductal adenocarcinoma (PDAC) is an aggressive malignancy characterized by abundant granulocytic myeloid-derived suppressor cells $\left(\mathrm{G}-\mathrm{MDSC}=\mathrm{CD} 45^{+} / \mathrm{Lin}^{-} / \mathrm{CD} 33^{+} / \mathrm{CD} 11 \mathrm{~b}^{+} / \mathrm{CD} 15^{+}\right)$, which infiltrate tumors and suppress anti-tumor immunity. We have previously demonstrated in a murine model of PDAC that zoledronic acid (ZA) depletes G-MDSC resulting in decreased tumor growth and improved survival. We report here the results of a phase 1 clinical trial (NCT00892242) using ZA as neo-adjuvant, perioperative therapy in patients with non-metastatic, resectable pancreatic adenocarcinoma. Methods: Eligible PDAC patients received ZA (4 mg) IV 2 weeks prior to surgery. Patients then received 2 additional doses of ZA 4 weeks apart. Blood and bone marrow were obtained from patients prior to treatment with ZA and 3 months after surgery for analysis of G-MDSC by flow cytometry. Results: Twenty-three patients received pre-operative ZA with at least 6 months of follow-up. Only 15 PDAC patients had nonmetastatic PDAC, which was amenable to resection. ZA was well tolerated, and all adverse events were grade 1 or 2 . The most common adverse events were fatigue, abdominal pain/discomfort, anorexia, and arthralgia. Of resected PDAC patients treated with ZA, 1- and 2-year overall survival (OS) was $85.7 \%$ and $33.3 \%$, respectively, with a median OS of 18 months. This group had a 1- and 2-year progression-free survival (PFS) of $26.9 \%$ and $8.9 \%$, respectively, with a median PFS of 12 months. The prevalence of G-MDSC was unchanged in the blood and bone marrow of PDAC patients pre- and post-treatment with ZA. Conclusion: ZA is safe and well tolerated as neo-adjuvant, peri-operative therapy in PDAC patients. In this small study, we did not observe a difference in OS or PFS compared to historical controls. Also, there was no difference in the prevalence of G-MDSC in the blood and bone marrow of PDAC patients pre- and posttreatment with ZA.
\end{abstract}

Keywords: Pancreatic Cancer; Zoledronic Acid; Myeloid-Derived Suppressor Cells

\section{Introduction}

Pancreatic ductal adenocarcinoma (PDAC) is a highly aggressive malignancy and the fourth leading cause of cancer death in the United States [1]. The prognosis of these patients is dismal with a 5-year overall survival less than $5 \%$ and a median survival of 6 months [1]. Even with complete surgical resection, recurrence is common and a majority of patients recur with distant metastasis. Patients who are able to undergo resection for early stage
PDAC have a 5-year survival of up to $25 \%$, with a median survival of 11 to 20 months [2,3]. Patients who develop recurrence usually present between 9 to 12 months after resection [4]. There is a clear need for novel therapies, as little progress has been made in the last several decades.

PDAC is characterized by a dense stroma with abundant immune cells, of which, myeloid-derived suppressor cells (MDSC) are predominant [5]. MDSC are a heterogeneous group of myeloid cells which suppress anti-tu- 
mor immunity and contribute to therapeutic resistance [6]. Therapies that ignore the stroma are likely to be ineffective in PDAC. We have previously described granulocytic-MDSC (G-MDSC) in human (CD45 $/ \mathrm{Lin}^{-} / \mathrm{CD} 33^{+} /$ $\left.\mathrm{CD} 11 \mathrm{~b}^{+} / \mathrm{CD} 15^{+}\right)$and murine $\left(\mathrm{CD} 45^{+} / \mathrm{CD} 11 \mathrm{~b}^{+} / \mathrm{Gr}^{\mathrm{hi}} / \mathrm{Ly} 6 \mathrm{G}^{+}\right)$ PDAC, and demonstrated their immunosuppressive and tumor-promoting properties [7]. These cells arise from the bone marrow in a process driven by the tumor. Depletion of MDSC in murine models has been associated with improved host immune responses resulting in decreased tumor growth, improved survival, and increased efficacy of vaccine therapy [8-10].

Zoledronic acid is a potent aminobisphosphonate typically used in the treatment/prevention of osteoporosis and pathologic fractures. ZA inhibits farnesyl-pyrophosphate-transferase which, in addition to its inhibitory effect on osteoclasts, has been shown to prevent tumormediated myelopoiesis associated with the generation of MDSC [10]. Although the mechanism of action in tumor bearing animals appears to involve blockade of hematopoiesis, hematological toxicities of the drug on normal bone marrow are generally not observed [11]. We have previously demonstrated that ZA depletes G-MDSC in a murine model of PDAC resulting in enhanced anti-tumor immunity, decreased tumor growth, and improved survival [7]. This encouraging preclinical data was the impetus for the current application of ZA in this clinical study.

Immunotherapy has made large strides in the treatment of human malignancy and will undoubtedly continue to do so. Strategies, such as anti-PD1 and anti-CTLA-4 therapies, are showing promise in cancer, with evidence of enhanced anti-tumor immunity and improvement in patient survival $[12,13]$. We present here the results of a Phase I clinical trial (NCT00892242) using ZA as neoadjuvant, peri-operative therapy in PDAC patients with nonmetastatic, resectable tumors. We sought to primarily establish the safety of ZA in this patient population, while also evaluating the effects of ZA on 1) overall survival (OS), 2) progression-free survival (PFS), and 3) the prevalence G-MDSC in the blood and bone marrow.

\section{Materials and Methods}

\subsection{Study Design}

This was a phase 1 study to determine safety and feasibility of perioperative ZA in patients with resectable PDAC (Figure 1). This study was approved by the Institutional Review Board and Human Research Protection Organization at our institution. All patients provided informed, written consent and were treated and Barnes-Jewish Hospital/Washington University Medical Center. Biopsyproven PDAC patients with tumors that appeared amenable to surgical resection based on pre-operative imaging were eligible for this study. Patients underwent blood draw and bone marrow biopsy at baseline and then received 4 mg IV of Zoledronic Acid (Zometa, Novartis) 2 weeks $+/-7$ days prior to surgery. Patients were then taken to the operating room with the intent to remove their tumor (pancreatectomy). Patients who underwent pancreatectomy received 2 additional $4 \mathrm{mg}$ IV doses of ZA at q4 week intervals (total of 3 doses). Three months after surgery, patients underwent an additional blood draw and bone marrow biopsy. The primary endpoint of the study was to evaluate the safety of neoadjuvant, perioperative ZA in PDAC patients. Secondary endpoints were: 1) overall survival; 2) progression-free survival; and 3) the prevalence G-MDSC in the blood and bone marrow pre- and posttreatment with ZA.

\subsection{Patients}

Patients with newly diagnosed, histologically or cytologically confirmed diagnosis of resectable pancreatic adenocarcinoma who were candidates for surgical treatment were eligible for this study. The eligibility criteria were defined as follows: measurable or evaluable disease defined by RECIST criteria; $>18$ years old; Karnofsky Performance Status (KPS) $\geq 70$; life expectancy $>12$ weeks; adequate bone marrow functions defined as an absolute neutrophil count $>1500 / \mathrm{mm}^{3}$, platelet count $>100,000 /$ $\mathrm{mm}^{3}$ and hemoglobin $>10 \mathrm{~g} / \mathrm{dL}$; adequate renal function defined as serum creatinine $\leq 1.3 \mathrm{mg} / \mathrm{dL}$ or creatinine clearance $\geq 90 \mathrm{mg} / \mathrm{min} / 1.73 \mathrm{~m}^{2}$ with a serum creatinine $>$ $1.3 \mathrm{mg} / \mathrm{dl}$; adequate hepatic function defined as total bilirubin $\leq 1.5 \times$ the institutional upper limit normal value (ULN) after relieving biliary obstruction and aspartate aminotransferase (AST) $\leq 2 \times$ the ULN. The following patients were excluded from the study: pregnant patients, patients with prior or current autoimmune disease, HIV+ patients, patients receiving other investigational drugs, patient treated with a bisphosphonate within the previous 6 month, patient with current active dental problems. A prospectively maintained database of all patients undergoing pancreaticoduodenectomy for PDAC at Barnes-Jewish Hospital from 1997 to 2012 was used to estimate survival of historical controls for survival comparison.

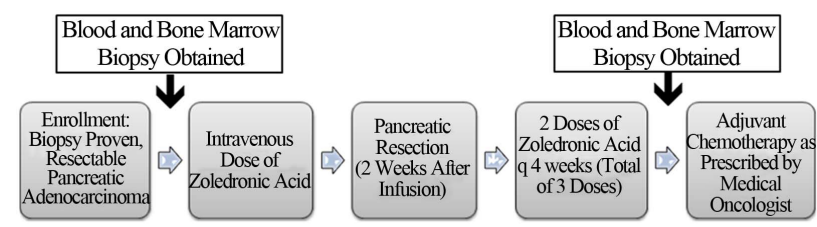

Figure 1. Flow diagram depicts the design of the clinical trial. 


\subsection{Safety Assessment}

Adverse events were monitored continuously during treatment. Toxicity was characterized according to the National Cancer Institute Common Terminology Criteria for Adverse Events version 3.0 (CTCAE). Follow up on subject well-being was performed by telephone on the first or second day after infusion. Subjects maintained a study diary for two weeks post infusion to note any symptoms related to ZA infusion.

\subsection{Efficacy Assessment}

All patients who received at least 1 does of ZA were followed up for survival. Patients followed up with a physician 1,3, and 6 months after surgery and routine labs were obtained at each visit. Although tumor response was not the primary endpoint of this trial, subjects were monitored for recurrence during the event monitoring period, as clinically indicated. Measurable disease was assessed by the Response Evaluation Criteria in Solid Tumor (RECIST) 1.1.

\subsection{Flow Cytometry on Blood and Bone Marrow Cells}

Peripheral blood samples and bone marrow aspirates (iliac crest) were collected from patients at baseline and 1 month following the last dose of ZA (3 months after surgery) in vacuum tubes containing lithium heparin (BD Biosciences; San Jose, CA). Cells were isolated by Ficoll-density centrifugation ((Ficol/Lite LymphoH, Atlanta Biologicals) and frozen in dimethyl sulfoxide (DMSO) with $10 \%$ fetal bovine serum (FBS) at $-80^{\circ} \mathrm{C}$. When both pre and post treatment blood samples were collected, PBMC and Bone marrow mononuclear cells were thawed using CTL wash (Cellular Technology Ltd, Shaker Heights, $\mathrm{OH}$ ) and washed. Cells were then $\mathrm{Fc}$ blocked (TruStain $\mathrm{FcX}^{\mathrm{TM}}$, Biolegend), stained, and fixed in according to manufacturers' protocol. Antibodies used included CD45 (AF700; Biolegend, HI30), CD11b (AF488; Biolegend, ICRF44), CD33 (PE; Biolegend, WM53), CD14 (APC/Cy7; Biolegend, M5E2), CD15 (Pacific Blue; Biolegend, W6D3), and HLA-DR (PE/Cy7; Biolegend, L243). Analysis was immediately performed on a LSR II flow cytometer (BD Biosciences; San Jose, CA). All results were analyzed using Flow Jo software (Tree Star Inc., Ashland, OR).

\subsection{Statistical Analysis}

All data was analyzed using GraphPad Prizm 5.01 software. Survival was estimated from the time of surgical resection using the Kaplan-Meier method. Survival was compared between 2 groups using the log-rank (Man-
tel-Cox) test. The prevalence in the blood and bone marrow of PDAC patients pre- and post-treatment with ZA were compared using paired t-tests. $\mathrm{P}<0.05$ was considered as statistically significant in all comparisons made.

\section{Results}

\subsection{Patient Data}

Twenty-three patients were enrolled in the trial, received at least one pre-operative dose of zoledronic acid (ZA), and had at least 6 months of follow-up after the first dose. These patients were taken to the operating room where seven $(30.4 \%)$ were found to have metastatic disease or locally advanced disease that precluded tumor resection. The remaining sixteen patients underwent successful pancreaticoduodenectomy (PD). One patient was discovered postoperatively to have benign disease upon pathology and was therefore excluded from the trial, leaving 15 patients with resected PDAC for analysis (Table 1). The median age of this group was 67 years (range 51 81 years) and the majority $(73.3 \%)$ was male. Ten of fifteen $(66.7 \%)$ patients required portal vein or superior mesenteric vein reconstruction at the time of surgery. All resected patients, coincidentally, had T3/N1/M0 disease (Stage 2B), and the median tumor size was $2.9 \mathrm{~cm}$ (range $1.2-5.5 \mathrm{~cm})$. All tumors were moderately $(8 / 15,53.3 \%)$ or poorly $(7 / 15,46.7 \%)$ differentiated, and seven $(46.7 \%)$

Table 1. Patient demographic and pathologic data.

\begin{tabular}{cc}
\hline Variable & No. \\
\hline Age & $67(51-81)$ \\
Median Years (Range) & $11(73.3 \%)$ \\
Sex & $4(26.7 \%)$ \\
Male (\%) & \\
Female (\%) & $2.9(1.2-5.5)$ \\
Tumor Size & \\
Median cm (Range) & $8(53.3 \%)$ \\
Histologic Grade & $7(46.7 \%)$ \\
Moderate (\%) & \\
Poor (\%) & $10(66.7 \%)$ \\
Vein Resection & $5(33.3 \%)$ \\
Performed (\%) & \\
Not Performed (\%) & $8(53.3 \%)$ \\
Resection Margin & $7(46.7 \%)$ \\
Negative (\%) & \\
Positive (\%) & \\
\hline
\end{tabular}

All patients had T3 N1 M0 disease (Stage 2B). 
had microscopically positive margins. Of resected patients, twelve $(80.0 \%)$ completed the ZA dosing regimen as designed; one patient $(6.7 \%)$ received only a single pre-operative dose and two patients (13.3\%) received one pre-operative and one post-operative dose. Post-ZA chemotherapy regimens are indicated in Table 2.

\subsection{Safety}

Generally, ZA was well tolerated in terms of toxicity. The majority of adverse events occurring in patients treated with ZA were consistent with findings commonly seen in most PDAC patients (Table 3). All adverse events were grade 1 or 2 , and there were no grade 3 or 4 events. The most common toxicity reported was fatigue $(\mathrm{n}=6)$. Like most patients with PDAC, gastrointestinal events composed the largest subclass of adverse events with abdominal pain/discomfort $(n=5)$, anorexia $(n=4)$, diarrhea $(n=2)$, and elevated liver-function tests $(n=2)$ being most common. The most common non-gastrointestinal adverse event was arthralgia $(n=4)$. No adverse events required scheduling or dosage adjustments of ZA.

\subsection{Clinical Efficacy}

Patients undergoing PD had a median overall survival of 18.0 months with 1- and 2-year overall survival of $85.7 \%$ and 33.3\%, respectively (Figure 2(a)). Comparatively, Stage 2B PDAC patients undergoing PD at our institution over the last 15 years $(n=355)$ had a median overall

Table 2. Post-zoledronic acid adjuvant therapy.

\begin{tabular}{cc}
\hline Treatment & Number of Patients \\
\hline Gemcitabine & 4 \\
5-Fluorouracil \& Radiation & 4 \\
Gemcitabine \& Erlotinib & 1 \\
Abraxane & 1 \\
Unknown & 5 \\
\hline
\end{tabular}

Table 3. Adverse events in $>5 \%$ of patients.

\begin{tabular}{cc}
\hline Adverse Events & Number of Patients \\
\hline Fatigue & $6(26.1 \%)$ \\
Abdominal Pain/Discomfort & $5(21.7 \%)$ \\
Anorexia & $4(17.4 \%)$ \\
Arthralgia & $4(17.4 \%)$ \\
Elevated LFTs & $2(8.7 \%)$ \\
Diarrhea & $2(8.7 \%)$ \\
\hline
\end{tabular}

Back pain, constipation, cough, dizziness, edema, headache, hypophosphatemia, neuropathy, pyrexia, and rigors were each reported by one patient.

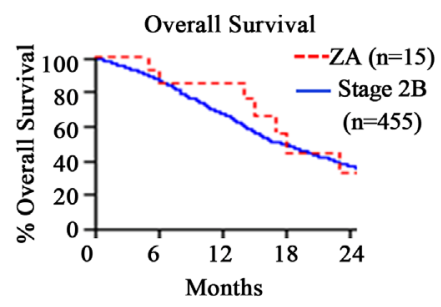

(a)

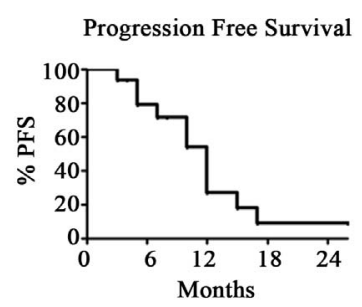

(b)

Figure 2. (a) Kaplan-Meier survival curves compare overall survival of patients treated with ZA (Red-dotted) to historical Stage 2B patients (Blue-solid); (b) Kaplan-Meier survival curve depicts progression-free survival (PFS) of patients treated with $\mathbf{Z A}$.

survival of 17.7 months with 1- and 2-year survivals of $67.0 \%$ and $37.4 \%$, respectively. Therefore, overall survival in this small cohort of patients treated with ZA was not significantly different from historical controls $(\mathrm{p}=$ 0.9404.) The median progression-free survival (PFS) in ZA treated patients was 12 months with 1- and 2-year PFS of $26.9 \%$ and $8.9 \%$, respectively (Figure 2(b)). At the time of this analysis, eleven patients had experienced recurrence of their cancer; four patients $(36.4 \%)$ had distant metastasis, five patients $(45.5 \%)$ experienced local recurrence, and in 2 patients $(18.2 \%)$ the recurrence site was unknown.

\subsection{Effect of ZA on G-MDSC in Blood and Bone Marrow}

We analyzed the blood and bone marrow of the 12 PDAC patients who completed the ZA regimen at baseline and 3 months after surgery for alterations in the prevalence G-MDSC by flow cytometry $\left(\mathrm{CD} 45^{+} / \mathrm{Lin}^{-} / \mathrm{CD} 33^{+} /\right.$ $\left.\mathrm{CD} 11 \mathrm{~b}^{+} / \mathrm{CD} 15^{+}\right)$. We detected no statistically significant difference in the prevalence of G-MDSC in the blood or bone marrow following treatment with ZA (Figure 3). Furthermore, neither pre- nor post-treatment G-MDSC prevalence predicted recurrence or survival in this small cohort of patients (data not shown).

\section{Discussion}

The purpose of this phase 1 study was to evaluate the safety and efficacy of peri-operative Zoledronic acid (ZA) in patients with resectable PDAC. In general, ZA was 


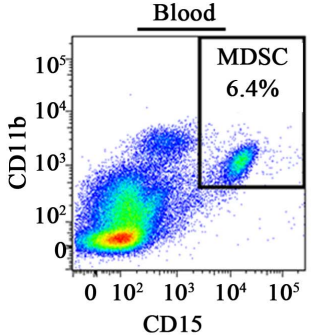

(a)

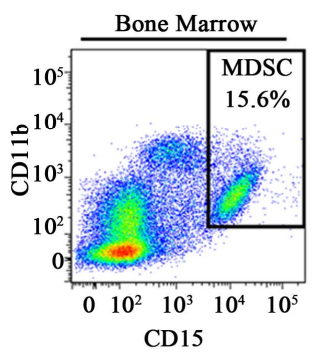

(c)

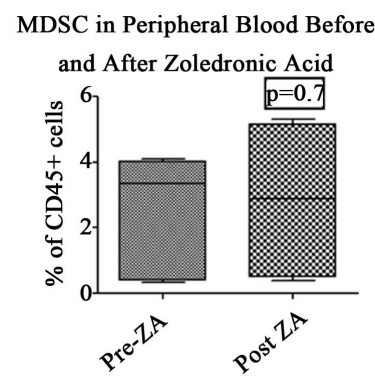

(b)

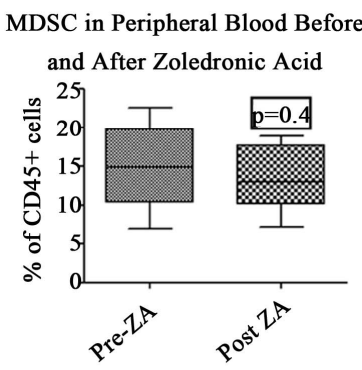

(d)
Figure 3. Peripheral blood and bone marrow mononuclear cells from PDAC patients were collected pre- and posttreatment with zoledronic acid (ZA) and flow cytometry was performed to compare changes in the prevalence of myeloid derived suppressor cells $\left(\mathrm{CD}^{+}, \mathrm{Lin}^{-}, \mathrm{CD33}^{+}\right.$, $\mathrm{CD}_{11 b^{+}}, \mathrm{CD}^{+} 5^{+}$). (a) Representative flow cytometry plot from blood of PDAC patient; (b) Graph depicts G-MDSC prevalence in the blood pre- and post-treatment; (c) Representative flow cytometry plot from bone marrow of PDAC patient; (d) Graph depicts G-MDSC prevalence in the bone marrow of PDAC patients pre- and post-treatment with $\mathrm{ZA}$. Graphs depict means \pm SEM. $p$ values are by paired t-tests.

well tolerated, and we observed no grade 3 or 4 toxicities in the 23 patients who received peri-operative treatment. These findings are consistent with the toxicity profiles using ZA in other diseases such as osteoporosis and prevention of pathologic fractures [14]. Therefore, we have established the safety of ZA in the neo-adjuvant, perioperative setting in PDAC patients. Future trials can be designed to further evaluate the appropriate application of this agent to PDAC in this setting.

This study is remarkable for the relative homogeneity with regard to the stage and grade of patients' tumors. The PDAC patients who underwent PD in our study had aggressive tumors, which were all T3 N1 (Stage 2B) and moderately or poorly differentiated. In addition, twothirds of patients required portal vein or superior mesenteric vein resection; a rate that is roughly three times the national average [15]. Furthermore, nearly half of these patients had microscopically positive resection margins, further indicating the invasive nature of the tumors in this cohort. We did not observe a difference in overall survival between the cohort of ZA-treated PDAC patients who underwent PD and historical Stage 2B PDAC patients treated at our institution. In addition to the small size of the cohort, a lack of standardized adjuvant therapy may have also made it difficult to detect a survival benefit in these patients compared to historical controls. Following surgery and ZA infusions, the decision with regards to administer further chemotherapy and/or radiation was at the discretion of the patient's medical oncologist. This could obviously confound patient outcome as the standard of care following surgical resection is currently a subject of debate [16].

Our group has previously demonstrated that treatment with ZA inhibits the expansion of G-MDSC (CD45 $\left.\mathrm{CD}_{11 \mathrm{~b}}, \mathrm{Gr}^{+}\right)$in murine PDAC [7]. A decrease in GMDSC at the site of tumor correlated with decreased tumor growth and improved survival in vivo. In addition, tumor-infiltrating regulatory $\mathrm{T}$ cells were also decreased while the number of immune effectors cells was increased. Similar to our preclinical findings with ZA in PDAC, aminobisphosphonates have been shown to inhibit the accumulation of MDSC in murine breast cancer by preventing tumor-induced myelopoiesis [10]. This finding may have important translational applications, as studies have demonstrated that ZA improves PFS and OS in postmenopausal breast cancer patients as adjuvant therapy [17-19]. These trials have sparked much interest in ZA as a potent immunomodulatory agent in human malignancy. In addition to its effects on myelopoiesis, ZA has also been demonstrated to have direct antiproliferative, antimetastatic, and proapoptotic effects on human pancreatic cancer cell lines [20]. We conducted the clinical trial presented here to investigate the potential role for Zometa in human PDAC.

In accordance with ZA's apparent lack of effect on OS and PFS in this small cohort of PDAC patients, we did not observe a difference in the prevalence of G-MDSC in the blood or bone marrow of PDAC patients following treatment. This would imply that ZA is not depleting G-MDSC. One explanation for this could be the duration and dose of ZA administered. Patients were given $4 \mathrm{mg}$ IV once 2 weeks prior to surgical resection followed by every four weeks for a total of 3 doses. Studies that have demonstrated efficacy in breast cancer have treated patients for as long as 5 years. Additionally, the $4 \mathrm{mg}$ dose of ZA used in this study may have been insufficient. Doses of $8 \mathrm{mg}$ have been used safely in patients with malignant bone metastases and doses as high as $16 \mathrm{mg}$ have been shown to be safe in humans $[11,14]$. Perhaps, increasing the dose and/or duration of ZA would improve efficacy in human PDAC.

In conclusion, this Phase I trial demonstrates that ZA is safe and well tolerated when administered to PDAC patients in the neo-adjuvant, peri-operative setting. We 
did not observe a difference in PFS or OS in patients treated with ZA compared to historical controls. Further study is needed to better understand the apparent discrepancies between the efficacy of ZA in mice and humans as this may reveal key differences in translating preclinical immunological studies into clinical trials.

\section{Acknowledgements}

DCL acknowledges funding from Novartis. DGD acknowledges support from the Lustgarten Foundation, V Foundation, Edward Mallinckrodt Jr. Award, the Cancer Research Foundation and Siteman Cancer Center Career Development Award. DCL and DGD acknowledge the Siteman Frontier Funds Team Science Award. DES, JBM, and MRP acknowledge funding from NCI grant T32 CA 009621.

\section{REFERENCES}

[1] A. Jemal, R. Siegel, J. Xu and E. Ward, "Cancer Statistics, 2010," CA: A Cancer Journal for Clinicians, Vol. 60, No. 5, 2010, pp. 277-300. doi:10.3322/caac.20073

[2] R. Delcore, F. J. Rodriguez, J. Forster, A. S. Hermreck and J. H. Thomas, "Significance of Lymph Node Metastases in Patients with Pancreatic Cancer Undergoing Curative Resection," The American Journal of Surgery, Vol. 172, No. 5, 1996, pp. 463-468. doi:10.1016/S0002-9610(96)00237-1

[3] A. Richter, M. Niedergethmann, J. W. Sturm, D. Lorenz, S. Post and M. Trede, "Long-Term Results of Partial Pancreaticoduodenectomy for Ductal Adenocarcinoma of the Pancreatic Head: 25-Year Experience," World Journal of Surgery, Vol. 27, No. 3, 2003, pp. 324-329. doi:10.1007/s00268-002-6659-Z

[4] H. M. Karpoff, D. S. Klimstra, M. F. Brennan and K. C. Conlon, "Results of Total Pancreatectomy for Adenocarcinoma of the Pancreas," Archives of Surgery, Vol. 136, No. 1, 2001, pp. 44-47. doi:10.1001/archsurg.136.1.44

[5] P. Goedegebuure, J. B. Mitchem, M. R. Porembka, M. C. Tan, B. A. Belt, A. Wang-Gillam, W. E. Gillanders, W. G. Hawkins and D. C. Linehan, "Myeloid-Derived Suppressor Cells: General Characteristics and Relevance to Clinical Management of Pancreatic Cancer," Current Cancer Drug Targets, Vol. 11, No. 6, 2011, pp. 734-751. doi:10.2174/156800911796191024

[6] D. I. Gabrilovich and S. Nagaraj, "Myeloid-Derived Suppressor Cells as Regulators of the Immune System," Nature Reviews Immunology, Vol. 9, No. 3, 2009, pp. 162174. doi: $10.1038 /$ nri2506

[7] M. R. Porembka, J. B. Mitchem, B. A. Belt, C. S. Hsieh, H. M. Lee, J. Herndon, W. E. Gillanders, D. C. Linehan and P. Goedegebuure, "Pancreatic Adenocarcinoma Induces Bone Marrow Mobilization of Myeloid-Derived Suppressor Cells Which Promote Primary Tumor Growth," Cancer Immunology, Immunotherapy: CII, Vol. 61, No. 9, 2012, pp. 1373-1385.
[8] B. Zhang, Y. Zhang, N. A. Bowerman, A. Schietinger, Y. X. Fu, D. M. Kranz, D. A. Rowley and H. Schreiber, "Equilibrium between Host and Cancer Caused by Effector T Cells Killing Tumor Stroma," Cancer Research, Vol. 68, No. 5, 2008, pp. 1563-1571. doi:10.1158/0008-5472.CAN-07-5324

[9] L. Yang, L. M. DeBusk, K. Fukuda, B. Fingleton, B. GreenJarvis, Y. Shyr, L. M. Matrisian, D. P. Carbone and P. C. Lin, "Expansion of Myeloid Immune Suppressor Gr+ $\mathrm{CD}_{1} 1 \mathrm{~b}^{+}$Cells in Tumor-Bearing Host Directly Promotes Tumor Angiogenesis," Cancer Cell, Vol. 6, No. 4, 2004, pp. 409-421. doi:10.1016/j.ccr.2004.08.031

[10] C. Melani, S. Sangaletti, F. M. Barazzetta, Z. Werb and M. P. Colombo, "Amino-Biphosphonate-Mediated MMP9 Inhibition Breaks the Tumor-Bone Marrow Axis Responsible for Myeloid-Derived Suppressor Cell Expansion and Macrophage Infiltration in Tumor Stroma," Cancer Research, Vol. 67, No. 23, 2007, pp. 11438-11446. doi:10.1158/0008-5472.CAN-07-1882

[11] Novartis Pharma, "Zometa Product Information," Author, Basel, 2013.

[12] J. R. Brahmer, S. S. Tykodi, L. Q. Chow, W. J. Hwu, S. L. Topalian, P. Hwu, C. G. Drake, L. H. Camacho, J. Kauh, K. Odunsi, et al., "Safety and Activity of Anti-PD-L1 Antibody in Patients with Advanced Cancer," The New England Journal of Medicine, Vol. 366, No. 26, pp. 24552465. doi:10.1056/NEJMoa1200694

[13] F. S. Hodi, S. J. O’Day, D. F. McDermott, R. W. Weber, J. A. Sosman, J. B. Haanen, R. Gonzalez, C. Robert, D. Schadendorf, J. C. Hassel, et al., "Improved Survival with Ipilimumab in Patients with Metastatic Melanoma," The New England Journal of Medicine, Vol. 363, No. 8, 2010, pp. 711-723. doi:10.1056/NEJMoa1003466

[14] J. R. Berenson, R. A. Vescio, L. S. Rosen, J. M. VonTeichert, M. Woo, R. Swift, A. Savage, E. Givant, M. Hupkes, H. Harvey, et al., "A Phase I Dose-Ranging Trial of Monthly Infusions of Zoledronic Acid for the Treatment of Osteolytic Bone Metastases," Clinical Cancer Research, Vol. 7, No. 3, 2001, pp. 478-485.

[15] J. M. Winter, J. L. Cameron, K. A. Campbell, M. A. Arnold, D. C. Chang, J. Coleman, M. B. Hodgin, P. K. Sauter, R. H. Hruban, T. S. Riall, et al., "1423 Pancreaticoduodenectomies for Pancreatic Cancer: A Single-Institution Experience," Journal of Gastrointestinal Surgery, Vol. 10, No. 9, 2006, pp. 1199-1210.

[16] B. G. Smaglo and M. J. Pishvaian, "Postresection Chemotherapy for Pancreatic Cancer," The Cancer Journal, Vol. 18, No. 6, 2012, 614-623. doi:10.1097/PPO.0b013e31827459d8

[17] H. Eidtmann, R. de Boer, N. Bundred, A. Llombart-Cussac, N. Davidson, P. Neven, G. von Minckwitz, J. Miller, N. Schenk and R. Coleman, "Efficacy of Zoledronic Acid in Postmenopausal Women with Early Breast Cancer Receiving Adjuvant Letrozole: 36-Month Results of the ZOFAST Study," Annals of Oncology: Official Journal of the European Society for Medical Oncology/ESMO, Vol. 21, No. 11, 2010, pp. 2188-2194.

[18] M. Gnant, B. Mlineritsch, H. Stoeger, G. Luschin-Eben- 
greuth, D. Heck, C. Menzel, R. Jakesz, M. Seifert, M. Hubalek, G. Pristauz, et al., "Adjuvant Endocrine Therapy plus Zoledronic Acid in Premenopausal Women with Early-Stage Breast Cancer: 62-Month Follow-Up from the ABCSG-12 Randomised Trial," The Lancet Oncology, Vol. 12, No. 7, 2011, pp. 631-641. doi:10.1016/S1470-2045(11)70122-X

[19] R. E. Coleman, H. Marshall, D. Cameron, D. Dodwell, R. Burkinshaw, M. Keane, M. Gil, S. J. Houston, R. J. Grieve, P. J. Barrett-Lee, et al., "Breast-Cancer Adjuvant
Therapy with Zoledronic Acid," The New England Journal of Medicine, Vol. 365, No. 15, 2011, pp. 1396-1405. doi:10.1056/NEJMoa1105195

[20] P. Tassone, P. Tagliaferri, C. Viscomi, C. Palmieri, M. Caraglia, A. D'Alessandro, E. Galea, A. Goel, A. Abbruzzese, C. R. Boland, et al., "Zoledronic Acid Induces Antiproliferative and Apoptotic Effects in Human Pancreatic Cancer Cells in Vitro," British Journal of Cancer, Vol. 88, No. 12, 2003, pp. 1971-1978. doi:10.1038/sj.bjc. 6600986 